\title{
ALDH16A1 is a differentially expressed gene in brain metastatic human breast cancer.
}

Shahan Mamoor, MS

${ }^{1}$ shahanmamoor@gmail.com

East Islip, NY USA

Metastasis to the brain is a clinical problem in patients with breast cancer ${ }^{1-3}$. We mined published microarray data ${ }^{4,5}$ to compare primary and metastatic tumor transcriptomes for the discovery of genes associated with brain metastasis in humans with metastatic breast cancer. We found that aldehyde dehydrogenase family 16, member 1, encoded by ALDH16A1, was among the genes whose expression was most different in the brain metastases of patients with metastatic breast cancer as compared to primary tumors of the breast. ALDH16A1 mRNA was present at increased quantities in brain metastatic tissues as compared to primary tumors of the breast. Expression of ALDH16A1 in primary tumors was significantly correlated with patient recurrence-free survival. Modulation of ALDH16A1 expression may be relevant to the biology by which tumor cells metastasize from the breast to the brain in humans with metastatic breast cancer.

$\underline{\text { Keywords: }}$ breast cancer, metastasis, brain metastases, central nervous system metastases, aldehyde dehydrogenase family 16, member 1, ALDH16A1, systems biology of breast cancer, targeted therapeutics in breast cancer. 
One report described a $34 \%$ incidence of central nervous system metastases in patients compared administration of either neratinib or trastuzumab in conjunction with paclitaxel demonstrated that in a randomized, controlled setting, in breast cancer patients treated with neratinib, not only was the incidence of central nervous system recurrence significantly lower, the time to central nervous system metastasis was significantly delayed as compared to patients administered trastuzumab ${ }^{6}$. The alarmingly high rate of central nervous system metastasis described, as well as data, both anecdotal ${ }^{2}$ and from a randomized, controlled setting ${ }^{6}$ illustrating that treatment with trastuzumab may be associated with these events demands an enhanced understanding of the transcriptional makeup of brain metastatic tissues to support identification of therapeutic targets, whether they are treatment related or not. We performed a global comparative analysis of primary and metastatic tumors in patients with brain metastatic breast cancer ${ }^{4,5}$. We discovered differential and increased expression of the gene encoding aldehyde dehydrogenase family 16, member 1, ALDH16A1, in brain metastatic tissues of patients with metastatic breast cancer.

\section{$\underline{\text { Methods }}$}

We used datasets GSE $10893^{4}$ and GSE $43837^{5}$ for this global differential gene expression analysis of brain metastatic breast cancer in conjunction with GEO2R. GSE10893 was generated using Agilent-011521 Human 1A Microarray G4110A technology with $n=11$ primary breast tumors and $n=4$ brain metastases from patients with breast cancer; analysis was performed using platform GPL885. GSE43837 was generated using Affymetrix Human X3P Array technology with $n=19$ primary tumors of the breast and $n=19$ brain metastases from patients with breast cancer; analysis was performed using platform GPL6480. The Benjamini and Hochberg method of $p$-value adjustment was used for ranking of differential expression but raw $p$-values were used to assess statistical significance of global differential expression. Log-transformation of data was auto-detected, and the NCBI generated category of platform annotation was used. A statistical test was performed to evaluate whether ALDH16A1 gene expression was significantly different between primary tumors of the breast and brain metastases in humans with breast cancer using a two-tailed t-test. For Kaplan-Meier survival analysis, we used the Kaplan-Meier plotter online tool $^{7}$ for correlation of ALDH16A1 mRNA expression levels with recurrence-free survival in $n=1764$ patients.

\section{$\underline{\text { Results }}$}

We performed global comparative transcriptome analysis of metastatic tumor tissues of patients with metastatic breast cancer using published microarray data ${ }^{4,5}$ to describe the transcriptional landscape of brain metastasis in human breast cancer in an unbiased fashion and for the discovery of novel therapeutic targets.

\section{ALDH16A1 is differentially expressed in the brain metastases of patients with brain metastatic breast cancer.}

Through blind, systems-level analysis of published microarray data ${ }^{4}$, we identified aldehyde dehydrogenase family 16 , member 1 , encoded by ALDH16A1, as a differentially expressed gene in the breast metastatic tissues of humans with breast cancer (Table 1). When 
sorting each of the genes expressed in brain metastases based on significance of difference as compared to primary tumors of the breast in patients with breast cancer, ALDH16A1 ranked 235 out of 17418 total transcripts (Chart 1), equating to $98.7 \%$ differential expression. Differential expression of ALDH16A1 in the brain metastases of patients with metastatic breast cancer was statistically significant (Chart $1 ; p=7.00 \mathrm{E}-04)$.

To attempt to validate differential transcriptome-wide differential expression of ALDH16A1 in brain metastases in human metastatic breast cancer, we queried a second

\section{ALDH16A1 is expressed at higher levels in the brain metastases of patients with metastatic breast cancer.}

We obtained exact mRNA expression levels for ALDH16A1, in primary tumors of the breast and in brain metastasis of patients with brain metastatic breast cancer to determine direction and statistical significance of change in ALDH16A1 expression in brain metastatic tissues. ALDH16A1 was expressed at higher levels in the brain metastases of patients with breast cancer as compared to primary tumors of the breast, and this difference was statistically significant (Figure $1 ; p=0.000964$ ).

\section{ALDH16A1 expression is significantly correlated with survival outcomes in human breast} cancer.

We performed Kaplan-Meier survival analysis ${ }^{8}$ in 1764 breast cancer patients in total, to evaluate whether ALDH16A1 tumor expression was correlated with survival outcomes in breast cancer. We observed a statistically significant correlation between primary tumor expression of ALDH16A1 and recurrence-free survival (RFS) in patients with breast cancer, in the lower survival quartile (Figure 2). Patients whose primary tumors expressed low levels of ALDH16A1 possessed median RFS of 24.07 months, while patients whose tumors expressed high levels of ALDH16A1 possessed median RFS of 39 months. This difference in RFS based on ALDH16A1 tumor expression in patients with breast cancer was statistically significant (Figure 2, Chart 3; logrank $p$-value: 3.2e-5; hazard ratio: 0.7 (0.59-0.83)).

Thus, by mining published microarray data ${ }^{4,5}$ in an unbiased and systematic fashion, we identified aldehyde dehydrogenase family 16, member 1, encoded by ALDH16A1, as among the genes whose expression was most different, transcriptome-wide, in the brain metastases of patients with breast cancer when compared to primary tumors of the breast; we observed 


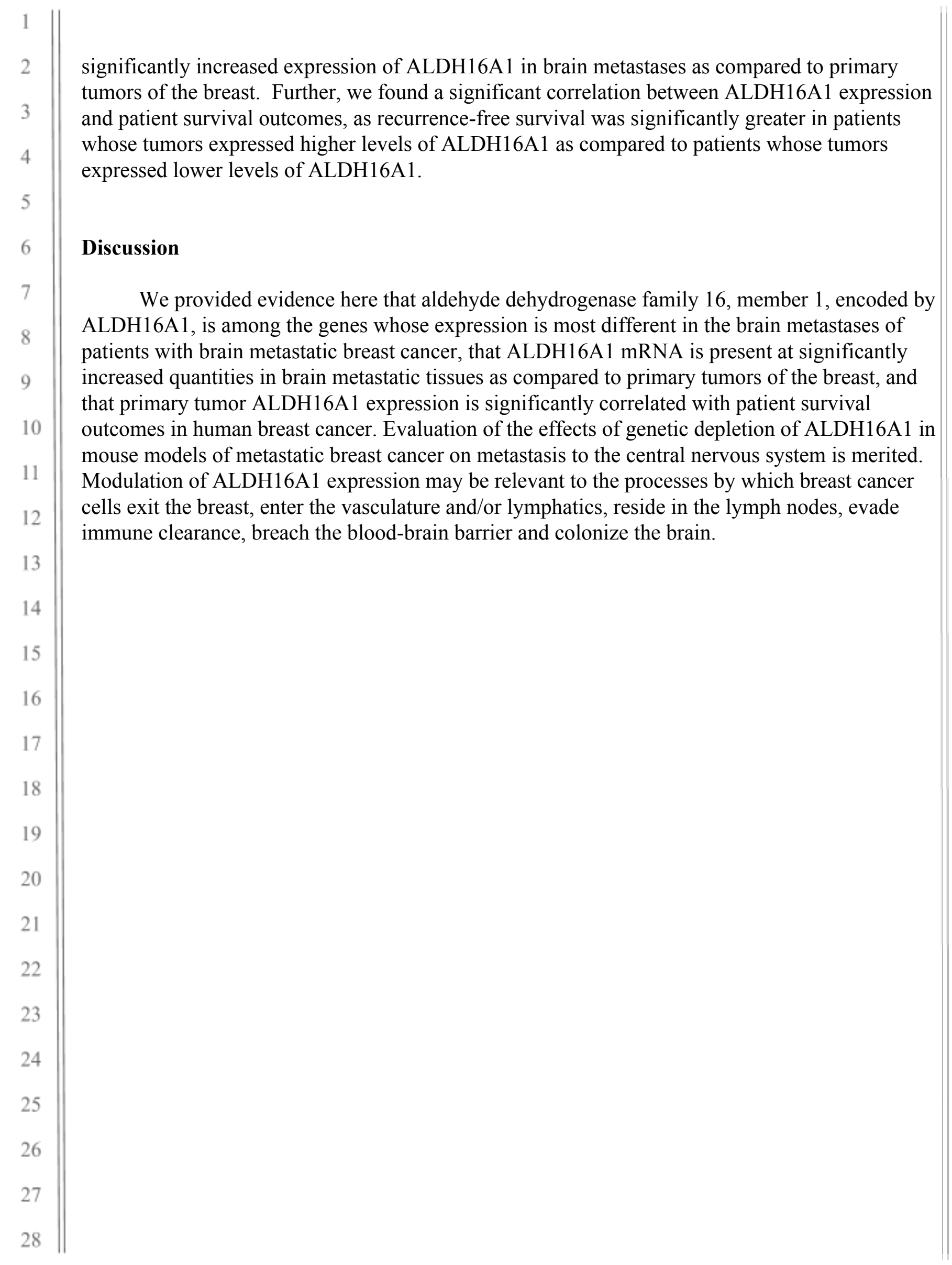




\section{References}

1. Lin, N.U., Amiri-Kordestani, L., Palmieri, D., Liewehr, D.J. and Steeg, P.S., 2013. CNS metastases in breast cancer: old challenge, new frontiers.

2. Bendell, J.C., Domchek, S.M., Burstein, H.J., Harris, L., Younger, J., Kuter, I., Bunnell, C., Rue, M., Gelman, R. and Winer, E., 2003. Central nervous system metastases in women who receive trastuzumab-based therapy for metastatic breast carcinoma. Cancer, 97(12), pp.2972-2977.

3. Tsukada, Y., Fouad, A., Pickren, J.W. and Lane, W.W., 1983. Central nervous system metastasis from breast carcinoma autopsy study. Cancer, 52(12), pp.2349-2354.

4. Weigman, V.J., Chao, H.H., Shabalin, A.A., He, X., Parker, J.S., Nordgard, S.H., Grushko, T., Huo, D., Nwachukwu, C., Nobel, A. and Kristensen, V.N., 2012. Basal-like Breast cancer DNA copy number losses identify genes involved in genomic instability, response to therapy, and patient survival. Breast cancer research and treatment, 133(3), pp.865-880.

5. McMullin, R.P., Wittner, B.S., Yang, C., Denton-Schneider, B.R., Hicks, D., Singavarapu, R., Moulis, S., Lee, J., Akbari, M.R., Narod, S.A. and Aldape, K.D., 2014. A BRCA1 deficient-like signature is enriched in breast cancer brain metastases and predicts DNA damage-induced poly (ADP-ribose) polymerase inhibitor sensitivity. Breast Cancer Research, 16(2), pp.1-10.

6. Awada, A., Colomer, R., Inoue, K., Bondarenko, I., Badwe, R.A., Demetriou, G., Lee, S.C., Mehta, A.O., Kim, S.B., Bachelot, T. and Goswami, C., 2016. Neratinib plus paclitaxel vs trastuzumab plus paclitaxel in previously untreated metastatic ERBB2-positive breast cancer: the NEfERT-T randomized clinical trial. JAMA oncology, 2(12), pp.1557-1564.

7. Györffy, B., Lanczky, A., Eklund, A.C., Denkert, C., Budczies, J., Li, Q. and Szallasi, Z., 2010. An online survival analysis tool to rapidly assess the effect of 22,277 genes on breast cancer prognosis using microarray data of 1,809 patients. Breast cancer research and treatment, 123(3), pp.725-731. 
Rank: 235

Probe ID: 1427

p-value: $7.00 \mathrm{E}-04$

t: -4.2

B: -0.263607

Gene: ALDH16A1

6 Gene name: aldehyde dehydrogenase family 16 , member 1

Chart 1: ALDH16A1 is differentially expressed in brain metastatic breast cancer when comparing brain metastases to primary tumors of the breast.

The rank of global differential expression, the probe/transcript ID, the $p$-value with respect to differential expression transcriptome-wide, $t$, a moderated $t$-statistic, $B$, the log-odds of differential expression between the groups compared, the gene and gene name are listed in this chart. 


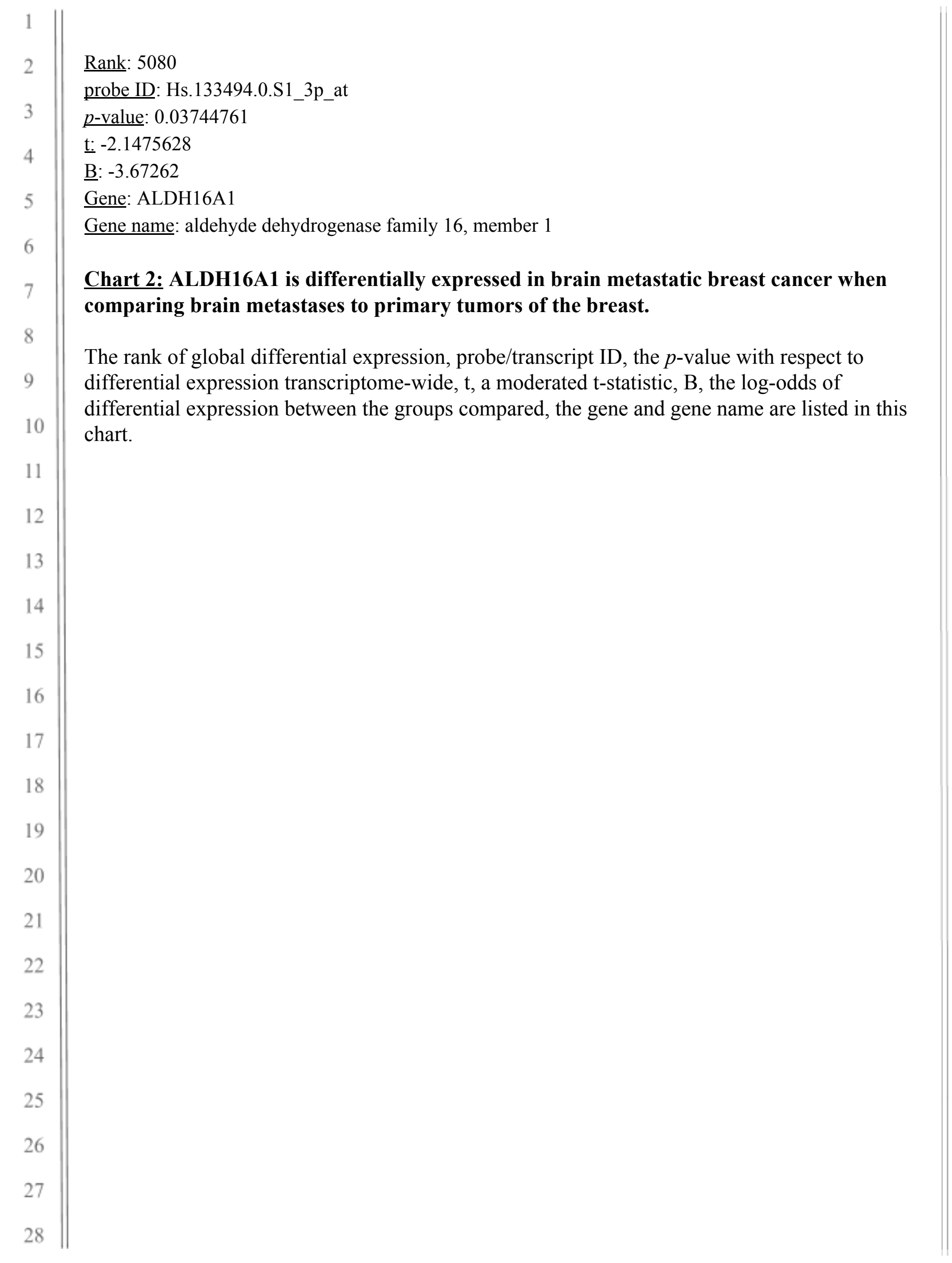




\section{ALDH16A1}

1.00

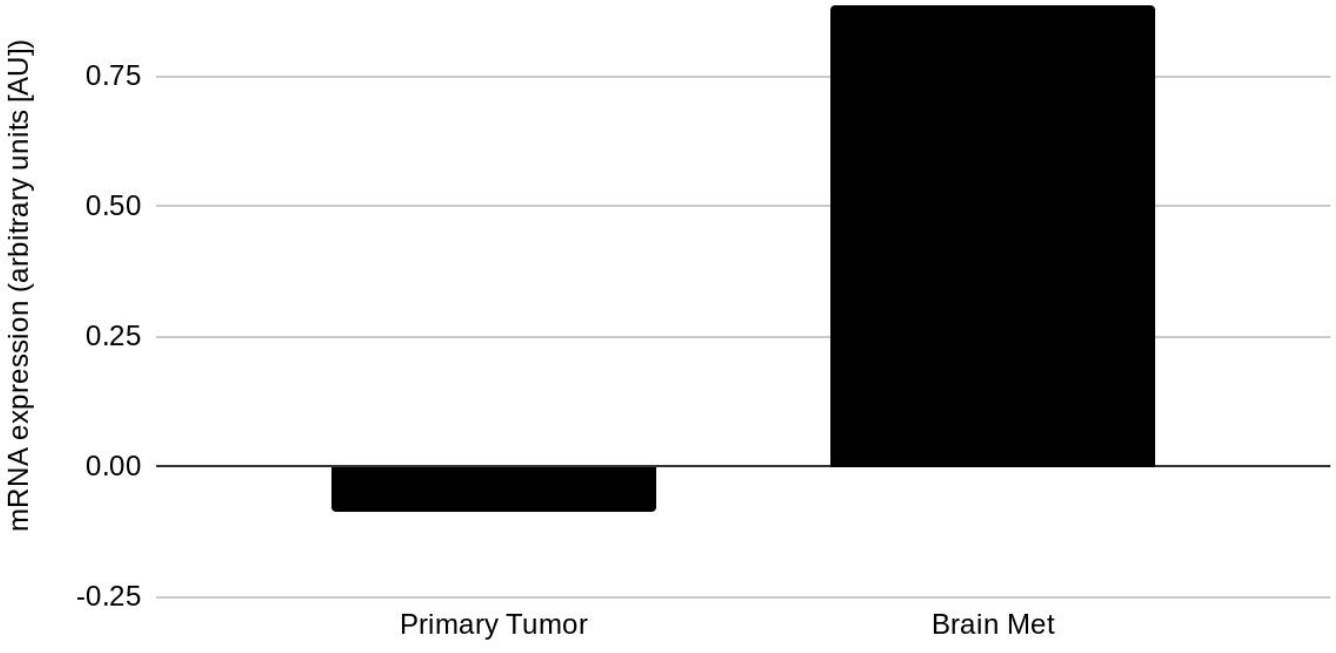

Figure 1: ALDH16A1 is expressed at significantly higher levels in the brain metastases of patients with metastatic breast cancer when compared to primary tumors of the breast.

The mRNA expression level of ALDH16A1 in primary tumors of the breast (left) and in brain metastases of women with metastatic breast cancer (right) is graphically depicted; the result of a statistical test evaluating significance of difference in ALDH16A1 expression between primary tumors of the breast and brain metastases is $p=0.000964$. 


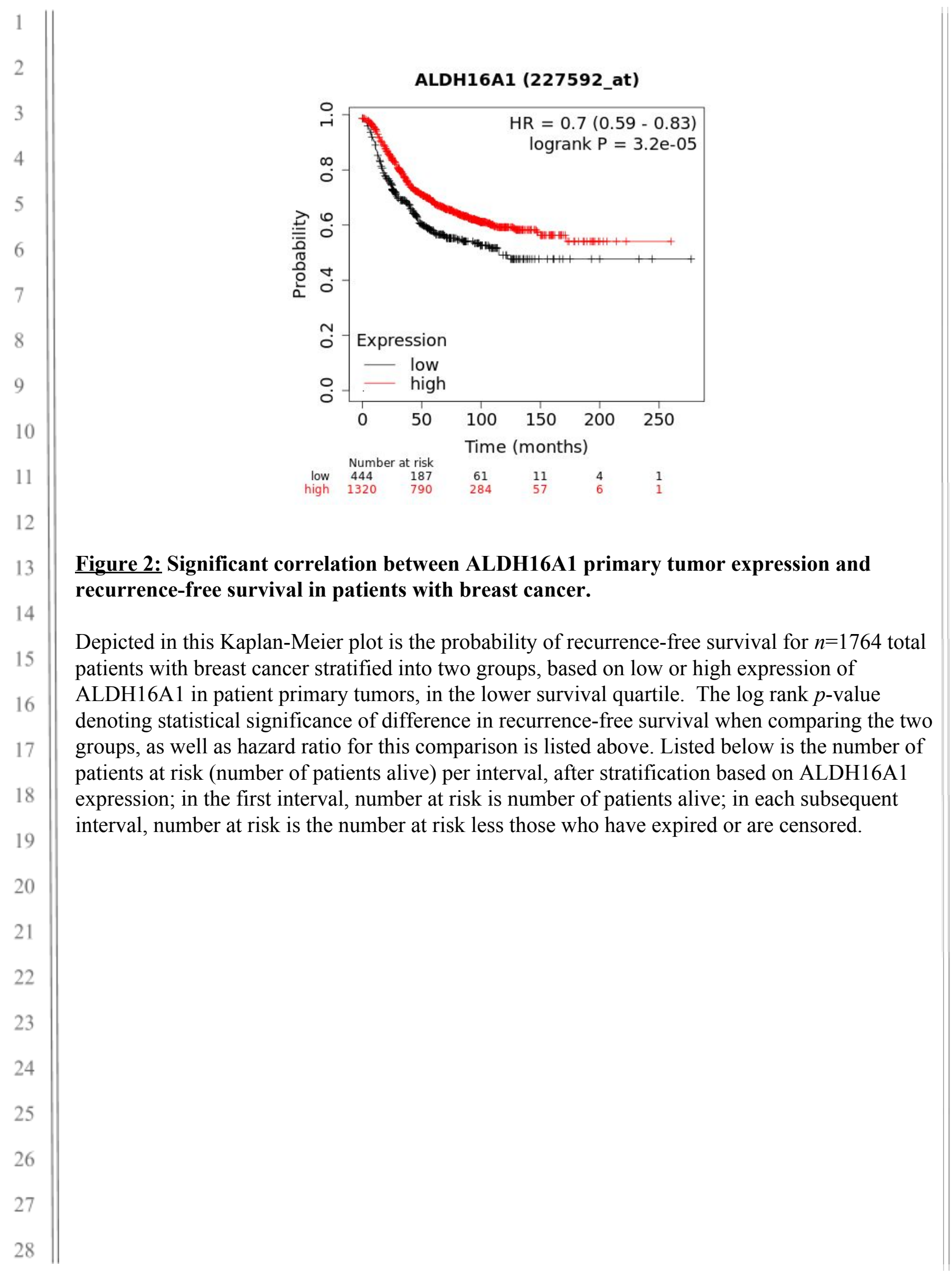




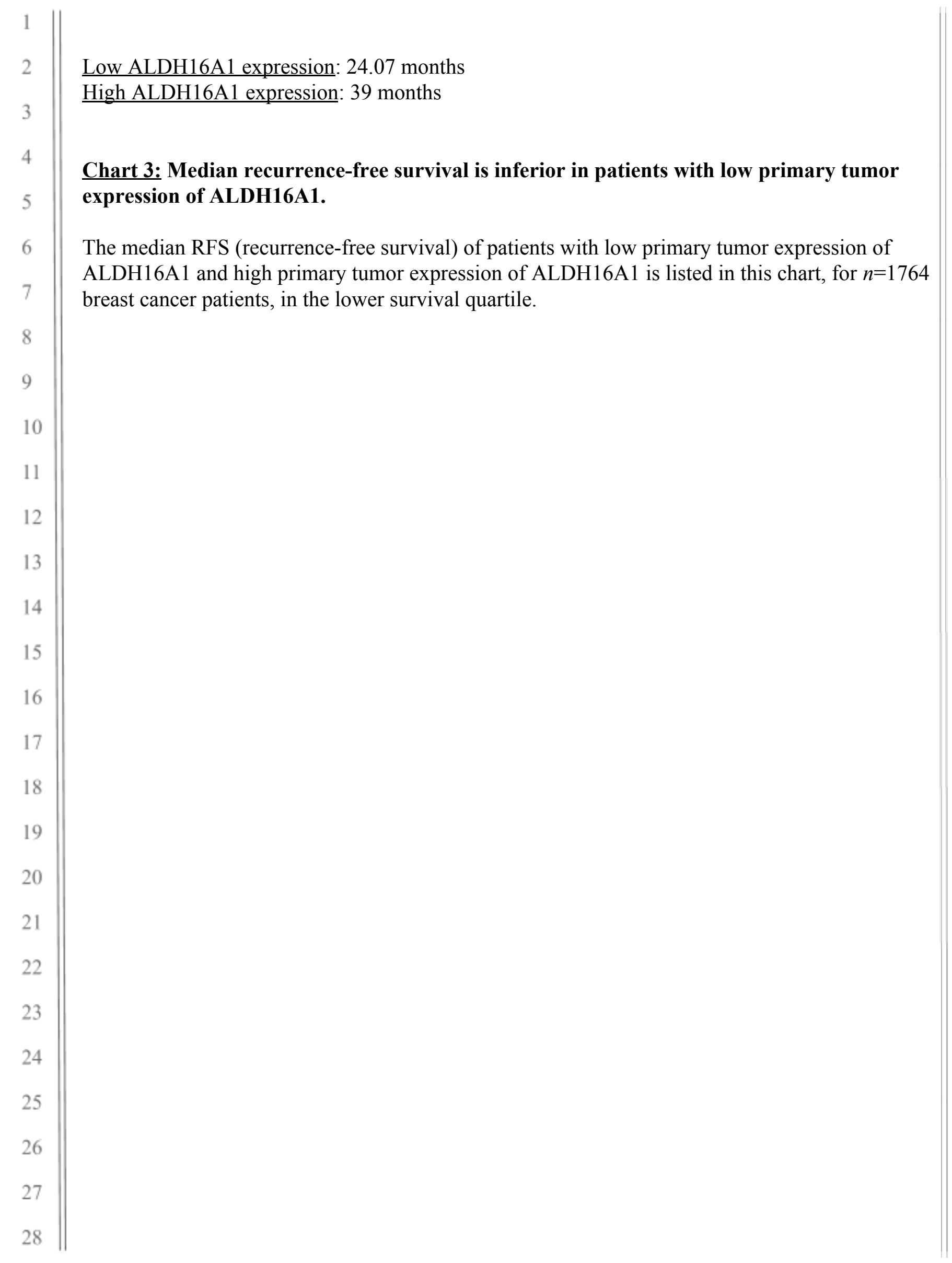

